This article is published with open access at Springerlink.com

\title{
Weak automorphisms of dihedral groups
}

\author{
ERNEST PŁONKA
}

\begin{abstract}
Let $\mathcal{A}=(A ; F)$ be an algebra with $T$ the set of all its term operations. For any permutation $\tau$ of $A$, the induced mapping $f \rightarrow \tau \circ f \circ \tau^{-1}$ defines a permutation $\tau^{\star}$ of the set of all finitary operations on the set A. We say that $\tau$ is a weak automorphism of $\mathcal{A}$ if and only if $\tau^{*}(T)=T$. Of course any automorphism $\alpha$ of $\mathcal{A}$ is a weak automorphism, because $\alpha^{*}(t)=t$ for all $t \in T$. The set of all weak automorphisms of $\mathcal{A}$ forms a subgroup of the symmetric group on $A$. In this paper, we describe weak automorphisms of the dihedral groups $\mathcal{D}_{n}$ for $n \geq 3$. We show that the weak automorphism group of $\mathcal{D}_{n}$ is a semidirect product of the group of automorphisms of $\mathcal{D}_{n}$ and some group related to the group of invertible elements of the ring $\mathbb{Z}_{n}$.
\end{abstract}

\section{Introduction}

The notion of a weak automorphism of a general algebra was introduced by A. Goetz in [1]. The first algebras in which this notion was studied were Boolean and Post algebras [13] and algebras having a basis [7]. Then some results were obtained for finite fields and rings [2,3] and mono-unary algebras [8]. Recently in $[11,12]$, the author has found a characterization of algebras whose weak automorphism groups are $k$-transitive for $k \geq 3$. In the case of groups, the image of the group operation $x \cdot y$ under the induced mapping $\tau^{\star}$ of a weak automorphism $\tau$ of a group $\mathcal{G}=\left(G ; \cdot,{ }^{-1}, 1\right)$ is a new group operation. One can ask under what conditions the mapping $\tau_{n}$ for $n \in Z$, defined by $\tau_{n}(g)=g^{n}$ for $g \in G$, is a weak automorphism of $\mathcal{G}$. This question was studied in $[9,14]$ for finite groups. The permutation $\tau_{-1}$ is a weak automorphism in any group. Clearly $\tau_{-1}^{\star}$ maps $x \cdot y$ onto $y \cdot x$. It is known that in abelian groups [4] and in the absolutely free groups [6], every weak automorphism is a superposition of an automorphism with $\tau_{-1}$ or simply an automorphism. There are some papers in which the authors are looking for group operations other than $x \cdot y$ or $y \cdot x$, which are not induced by a weak automorphism. In [5], nilpotent groups of class 2 were studied and in [10] such group operations were found in nilpotent groups of class 3 and 4 . In this paper, we determine weak automorphisms of dihedral groups $\mathcal{D}_{n}$ for $n \geq 3$, and we give a complete description of the structure of the group of weak automorphisms of $\mathcal{D}_{n}$. It turns out that this group is a semidirect product of the automorphism group

Presented by K. Kaarli.

Received May 9, 2009; accepted in final form September 9, 2009.

2000 Mathematics Subject Classification: Primary 08A40; Secondary 20F28, 08A35.

Key words and phrases: weak automorphism, dihedral group, group words. 
of $\mathcal{D}_{n}$ and some subgroup of the symmetric group on $D_{n}$ closely related to the group of invertible elements of the ring $\mathbb{Z}_{n}$.

\section{Some properties of weak automorphisms}

Let $\mathcal{A}=(A ; F)$ be a finitary algebra with $T^{(n)}$ the set of all $n$-ary term operations. A permutation $\tau$ of the set $A$ is said to be a weak automorphism of $\mathcal{A}$ if for all $n=0,1,2, \ldots$, the induced mapping $\tau^{\star}: A^{A^{n}} \rightarrow A^{A^{n}}$ defined by

$$
\left(\tau^{\star} f\right)\left(a_{1}, a_{2}, \ldots, a_{n}\right)=\tau\left(f\left(\tau^{-1}\left(a_{1}\right), \tau^{-1}\left(a_{2}\right), \ldots, \tau^{-1}\left(a_{n}\right)\right)\right.
$$

for $a_{1}, a_{2}, \ldots, a_{n} \in A$, transforms each set $T^{(n)}$ onto itself. This means that for every term $t \in T^{n}$ for $n=0,1, \ldots$, there exist two terms $t_{1}, t_{2} \in T^{n}$ such that $\tau^{\star}(t)=t_{1}$ and $\tau^{\star}\left(t_{2}\right)=t$ or, what is the same, the equalities

$$
\begin{aligned}
\tau\left(t\left(a_{1}, a_{2}, \ldots, a_{n}\right)\right) & =t_{1}\left(\tau\left(a_{1}\right), \tau\left(a_{2}\right), \ldots, \tau\left(a_{n}\right)\right), \\
\tau\left(t_{2}\left(a_{1}, a_{2}, \ldots, a_{n}\right)\right) & =t\left(\tau\left(a_{1}\right), \tau\left(a_{2}\right), \ldots, \tau\left(a_{n}\right)\right)
\end{aligned}
$$

hold for all $a_{1}, a_{2}, \ldots, a_{n} \in A$. The elements of the set $W A U T(\mathcal{A})$ of all weak automorphisms of an algebra $\mathcal{A}$ form a subgroup of the symmetric group $\left(S_{A} ; \circ,^{-1}, I d\right)$ of the set $A$, which contains the automorphism group $\operatorname{AUT}(\mathcal{A})$ as a normal subgroup.

In the case of a group $\mathcal{G}=\left(G ; \cdot,^{-1}, 1\right)$, the set $T^{(n)}$ consists of all words $w$ in $n$ variables of the form

$$
w\left(x_{1}, x_{2}, \ldots, x_{n}\right)=x_{i_{1}}^{n_{1}} x_{i_{2}}^{n_{2}} \cdots x_{i_{k}}^{n_{k}},
$$

where $1 \leq i_{1}, i_{2}, \ldots, i_{k} \leq n$ and $n_{1}, n_{2}, \ldots, n_{k} \in \mathbb{Z}$.

As a simple consequence of the definition of a weak automorphism, we get

Proposition 2.1. Let $\mathcal{G}=\left(G ; \cdot,{ }^{-1}, 1\right)$ be a group, and let $\tau$ be a permutation of the set $G$. Then $\tau$ is a weak automorphism of $\mathcal{G}$ if and only if

(1) $\tau(1)=1$,

(2) $\tau\left(g^{-1}\right)=(\tau(g))^{-1}, g \in G$,

(3) there exist two words $u(x, y)$ and $v(x, y)$ such that equalities

$$
\tau(g \cdot h)=u(\tau(g), \tau(h)) \quad \text { and } \quad \tau(v(g, h))=\tau(g) \cdot \tau(h)
$$

hold for all $g, h \in G$.

Proof. Routine.

Theorem 2.2. Let $\mathcal{G}=\left(G ; \cdot,^{-1}, 1\right)$ be a group. Suppose that $\tau$ is a weak automorphism of $\mathcal{G}$ such that $\tau^{\star}(x \cdot y)=w(x, y)=x \circ y$ for some word $w(x, y)$; that is, the equality

$$
\tau(g \cdot h)=\tau(g) \circ \tau(h)
$$

holds for all $g, h \in G$. The following equalities hold for all $g, h, k \in G$ and all integers $n$ :

(1) $\tau(1)=1$, 
(2) $1 \circ g=g \circ 1=g$,

(3) $(g \circ h) \circ k=g \circ(h \circ k)$,

(4) $g^{-1} \circ g=g \circ g^{-1}=1$,

(5) $\tau\left(g^{n}\right)=(\tau(g))^{n}$.

Therefore $\left(G ; \circ,{ }^{-1}, 1\right)$ is a group and $\tau$ is an isomorphism between the groups $\mathcal{G}$ and $(G ; \circ,-1,1)$.

Proof. We have $\tau(1)=1$, because $T^{(0)}=\{1\}$. This together with (2.1) gives

$$
1 \circ \tau(g)=\tau(1) \circ \tau(g)=\tau(1 \cdot g)=\tau(g)=\tau(g \cdot 1)=\tau(g) \circ \tau(1)=\tau(g) \circ 1,
$$

which proves (2). We have also

$$
\begin{aligned}
(g \circ h) \circ k & =\tau\left(\left(\tau^{-1}(g) \cdot \tau^{-1}(h)\right) \cdot \tau^{-1}(k)\right) \\
& =\tau\left(\tau^{-1}(g) \cdot\left(\tau^{-1}(h) \cdot \tau^{-1}(k)\right)\right)=g \circ(h \circ k) .
\end{aligned}
$$

Further, $\tau(g) \circ \tau\left(g^{-1}\right)=\tau\left(g \cdot g^{-1}\right)=1=\tau\left(g^{-1} \cdot g\right)=\tau\left(g^{-1}\right) \circ \tau(g)$, which means that $\tau\left(g^{-1}\right)$ is the inverse of the element $\tau(g)$ with respect to the operation $\circ$ and (4) follows. Now by easy induction on positive $n$ together with (4), we get (5), as required.

\section{Notations and some properties of dihedral groups}

Let $\mathcal{G}=\left(G ; \cdot,^{-1}, 1\right)$ be a group. Suppose that $\mathcal{H}$ is an arbitrary subgroup and $\mathcal{K}$ is a normal subgroup of $\mathcal{G}$. If each element $g$ from $G$ has the unique presentation $g=h \cdot k$ where $h \in H$ and $k \in K$, then the group $\mathcal{G}$ is said to be a semidirect product of $\mathcal{K}$ and $\mathcal{H}$. Denote $Z_{n}=\{0,1,2, \ldots, n-1\}$, and let $Z_{n}^{\times}$ stand for the set of all invertible elements of the ring $\mathbb{Z}_{n}=\left(Z_{n} ;+, \cdot,-, 0,1\right)$. For $n \geq 3$, the dihedral group $\mathcal{D}_{n}$ is a semidirect product of the group $\left(\{1,-1\} ; \cdot,^{-1}, 1\right)$ and $\left(Z_{n} ;+,-, 0\right)$, where $\varepsilon \in\{-1,1\}$ acts on $Z_{n}$ as the natural automorphism, $i \rightarrow \varepsilon \cdot i$ for $i \in Z_{n}$, of the group $\mathcal{Z}_{n}^{+}=\left(Z_{n} ;+,-, 0\right)$. Thus any element of the group $\mathcal{D}_{n}$ can be presented as a pair $(\varepsilon, i)$, where $\varepsilon \in\{1,-1\}$ for $i \in Z_{n}$. The operations in $\mathcal{D}_{n}$ are given by the formulas

$$
(\varepsilon, i) \cdot(\eta, j)=(\varepsilon \cdot \eta, \eta \cdot i+j), \quad(\varepsilon, i)^{-1}=(\varepsilon,-\varepsilon i), \quad 1=(1,0) .
$$

We use the standard notations:

$$
x^{-1} y x=y^{x}, \quad[y, x]=y^{-1} x^{-1} y x, \quad x^{\alpha+\beta y+z}=x^{\alpha}\left(x^{\beta}\right)^{y} x^{z}, \quad x^{\beta y}=\left(x^{y}\right)^{\beta},
$$

for arbitrary group elements $x, y, z$ and all integers $\alpha, \beta$. The following equalities are identities in any group:

$$
[y, x]^{-1}=[x, y], \quad[x y, z]=[x, z]^{y}[y, z], \quad[x, y z]=[x, z][x, y]^{z} .
$$

Let $\exp (\mathcal{G})$ denote the exponent of $\mathcal{G}$; that is, the smallest positive integer $t$ such that $g^{t}=1$ for all $g \in G$. 
Proposition 3.1. (i) For all elements $x=(\varepsilon, i), y=(\eta, j)$ from $D_{n}$ we have

$$
\begin{aligned}
{[y, x] } & =(1,(1-\eta) i+(\varepsilon-1) j), \\
{[y, x]^{x} } & =(1,(1-\eta) \varepsilon i+(1-\varepsilon) j), \\
{[y, x]^{y} } & =(1,(\eta-1) i+(\varepsilon-1) \eta j), \\
{[y, x]^{x y} } & =(1,(\eta-1) \varepsilon i+(1-\varepsilon) \eta j),
\end{aligned}
$$

and therefore the equation

$$
[y, x]^{x y}=[y, x]^{-1-x-y}
$$

is an identity in $\mathcal{D}_{n}$.

(ii) The equalities

$$
[[y, x],[u, v]]=1, \quad\left[x^{2}, y^{2}\right]=1, \quad\left[x^{2},[y, z]\right]=1, \quad U^{(1-x)(1+x)}=1,
$$

where $U$ is any product of commutators, are identities in $\mathcal{D}_{n}$.

Proof. This follows immediately from the definition of the group $\mathcal{D}_{n}$.

Proposition 3.2. Any word $w(x, y)$ of two variables $x, y$ in the group $\mathcal{D}_{n}$ can be written as

$$
w(x, y)=x^{s} y^{t}[y, x]^{A+B x+C y},
$$

where $s, t \in Z_{n}$ and $A, B, C \in Z_{e}$ for $e=\exp \left(\mathcal{D}_{n}^{\prime}\right)$.

Proof. Since the group $\mathcal{D}_{n}$ is of finite exponent, we can assume that $w(x, y)=$ $z_{1} \cdot z_{2} \cdots z_{k}$, where $z_{1}, z_{2}, \ldots, z_{k} \in\{x, y\}$. Using (3.1), we can transpose all $x$ 's which follow after $y$ 's. After this process, we obtain a word of the form $w^{\prime}=x^{t} u$, where $u$ is a product of $y$ 's and powers $[y, x]^{p(x)}$ of commutators for some polynomials $p(x)$. Now we remove all $y$ 's on the second place after $x^{t}$. According to (ii) of Proposition 3.1, all commutators and all squares of elements commute with each other, and therefore we can rewrite all products of commutators as $[y, x]^{q(x, y)}$ for some polynomial $q(x, y)$, which by $(3.2)$ is of the required form.

\section{Weak automorphisms of $\mathcal{D}_{n}$}

We begin with the following

Theorem 4.1. Let us define

$$
\tau_{A, a, b}(1, i)= \begin{cases}(1, a i) & \text { if } \varepsilon=1, i \in Z_{n}, \\ (-1, b+(1-2 A) a i) & \text { if } \varepsilon=-1, i \in Z_{n},\end{cases}
$$

where $a \in Z_{n}^{\times}, b \in Z_{n}$, and $A$ is an element of $Z_{n}$ such that $1-2 A \in Z_{n}^{\times}$. Then $\tau_{A, a, b}=\tau$ is a weak automorphism of the group $\mathcal{D}_{n}$ such that

$$
\begin{gathered}
\tau(g h)=\tau(g) \tau(h)[\tau(h), \tau(g)]^{A+B(\tau(g)+\tau(h))}, \\
\tau\left(g h[h, g]^{A_{1}+B_{1}(g+h)}\right)=\tau(g) \tau(h)
\end{gathered}
$$


hold for every $g, h \in D_{n}$, where

$$
B=(1-2 A)^{-1}\left(A-A^{2}\right)=-B_{1}, \quad A_{1}=-A(1-2 A)^{-1} .
$$

Proof. First of all observe that $\tau_{A, a, b}=\tau$ is a permutation of the set $D_{n}$. According to Proposition 2.1, we need only to check the equalities (4.1) and $(4.2)$.

Let $g=(\varepsilon, i)$ and $h=(\eta, j)$ for $\varepsilon, \eta \in\{-1,1\}$ and $i, j \in Z_{n}$. In view of Proposition 3.1, we have

$$
\begin{aligned}
{[y, x]^{A+B g+B y} } & =[y, x]^{A}\left([y, x]^{g}\right)^{B}\left([y, x]^{y}\right)^{B} \\
& =(1, i(1-\eta)(A+B(\varepsilon-1))+j(\varepsilon-1)(A+B(\eta-1))
\end{aligned}
$$

Using this, we can compare the left $L(g, h)$ and the right hand side $R(g, h)$ of the equation (4.1). We have

$$
\begin{aligned}
L((1, i),(1, j)) & =(1, a(i+j))=R((1, i),(1, j)), \\
L((1, i),(-1, j)) & =(-1, b+a(j-i)(1-2 A))=R((1, i),(-1, j)), \\
L((-1, i),(1, j) & =(-1, b+a(i+j)(1-2 A))=R((-1, i),(1, j)), \\
L((-1, i),(-1, j)) & =(1, a(j-i)), \\
R((-1, i),(-1, j)) & =\left(1, a(j-i)\left(1-4\left(A-A^{2}+2 A B-B\right)\right) .\right.
\end{aligned}
$$

Thus for $B$ defined in (4.3), the equation $L(g, h)=R(g, h)$ holds for all $g, h \in$ $D_{n}$.

Similarly, let $L(g, h)$ and $R(g, h)$ denote the left and the right hand side of the equation (4.2), respectively. We have

$$
\begin{aligned}
L((1, i),(1, j)) & =(1,(i+j) a)=R((1, i),(1, j)), \\
L((1, i),(-1, j)) & =\left(-1, b+i a(1-2 A)\left(2 A_{1}-1\right)+j a(1-2 A),\right. \\
R((1, i),(-1, j)) & =(-1, b-i a+j a(1-2 A)), \\
L((-1, i),(1, j)) & =\left(-1, b+i a(1-2 A)+j a(1-2 A)\left(1-2 A_{1}\right),\right. \\
R((-1, i),(1, j)) & =(-1, b+i a(1-2 A)+a j), \\
L((-1, i),(-1, j)) & =\left(1,(j-i) a\left(1-2 A_{1}+4 B_{1}\right)\right), \\
R((-1, i),(-1, j)) & =(1,(j-i) a(1-2 A)) .
\end{aligned}
$$

This means that for $A_{1}$ and $B_{1}$ which satisfy (4.3), the equality $L(g, h)=$ $R(g, h)$ holds for arbitrary choice of $\varepsilon, \eta$ and $i, j$. Therefore statement (4.2) is true, and consequently, $\tau_{A, a, b}$ is a weak automorphism of the group $\mathcal{D}_{n}$.

Theorem 4.2. Each weak automorphism of the group $\mathcal{D}_{n}$ is of the form $\tau_{A, a, b}$ for some triple of elements $A, a$, and $b$ from $Z_{n}$ such that $1-2 A, a \in Z_{n}^{\times}$.

Proof. Let $\sigma$ be a weak automorphism of $\mathcal{D}_{n}$ such that $\sigma^{*}(x \cdot y)=u(x, y)$ for some term $u$. By Proposition 3.2, we can assume $u(x, y)=x y[y, x]^{A+B x+C y}$ for some $A, B$, and $C$ from $Z_{n}$. Therefore the equality

$$
\sigma(g h)=\sigma(g) \sigma(h)[\sigma(h), \sigma(g)]^{A+B \sigma(g)+C \sigma(h)}
$$


holds for all $g, h \in D_{n}$. Since all elements of order $n$ belong to the set $X=$ $\left\{(1, i): i \in Z_{n}\right\}$, the element $\sigma(1,1)$ has to belong to $X$. By Theorem 2.2, we have $\sigma(1,1)=(1, a)$ for some $a \in Z_{n} \backslash\{0\}$. By (4.4) and induction on $i$, we obtain $\sigma(1, i)=\sigma(1, a i)$ for all $i \in Z_{n}$, and therefore $a \in Z_{n}^{\times}$. Suppose that $\sigma(-1,0)=(-1, b)$ for some $b \in Z_{n}$. Using the equality $(-1, j)=(-1,0) \cdot(1, j)$ and (4.4), we have

$$
\begin{aligned}
\sigma(-1, j) & =\sigma((-1,0) \cdot(1, j))=u((-1, b),(1, a j)) \\
& =(-1, b+j a(1-2(A-B+C))),
\end{aligned}
$$

for arbitrary $j \in Z_{n}$. Observe that $1-2(A-B+C)$ must be invertible in $\mathbb{Z}_{n}$, because otherwise $\sigma$ would not be a permutation of $D_{n}$. By putting $A^{\prime}=A-B+C$, we get $\sigma(\varepsilon, i)=\tau_{A^{\prime}, a, b}(\varepsilon, i)$ for $\varepsilon \in\{-1,1\}$ and all $i \in Z_{n}$, and the proof is complete.

Lemma 4.3. We have

$$
\begin{aligned}
\tau_{A, a, b}^{-1} & =\tau_{\perp A, a^{-1},-(1-2 A)^{-1} a^{-1} b,}, \\
\tau_{A, a, b} \circ \tau_{A^{\prime}, a^{\prime}, b^{\prime}} & =\tau_{A \dot{+} A^{\prime}, a a^{\prime},(1-2 A) a^{\prime} b+b^{\prime}}, \\
\tau_{A, a, b}^{-1} \circ \tau_{0, a^{\prime}, b^{\prime}} \circ \tau_{A, a, b} & =\tau_{0, a^{\prime},(1-2 A) a b^{\prime}},
\end{aligned}
$$

where $-A=-A(1-2 A)^{-1}$ and $A \dot{+} A^{\prime}=A+A^{\prime}-2 A A^{\prime}$.

Proof. This follows immediately from the definition of $\tau_{A, a, b}$.

\section{The group $W A U T\left(\mathcal{D}_{n}\right)$}

Now we are going to describe the structure of the group $\operatorname{WAUT}\left(\mathcal{D}_{n}\right)$. To do this we need an auxiliary result.

Lemma 5.1. The elements of the set

$$
Z_{n}^{\dot{+}}=\left\{A \in Z_{n}:(1-2 A) \in Z_{n}^{\times}\right\}
$$

form a group $\mathcal{Z}_{n}^{\dot{+}}=\left(Z_{n}^{\dot{+}} ; \dot{+},-\dot{-}, 0\right)$ with respect to the operations $\dot{+}$ and - given by the following formulas

$$
A \dot{+} B=A+B-2 A B, \quad \div A=-A(1-2 A)^{-1} .
$$

Proof. We check

$$
(A \dot{+} B) \dot{+} C=A+B+C-2 A B-2 A C-2 B C+4 A B C=A \dot{+}(B \dot{+} C),
$$

and also

$$
A \dot{+}(-A)=A+\left(-A(1-2 A)^{-1}\right)-2 A\left(-A(1-2 A)^{-1}\right)=0 .
$$

Let us observe that if $1-2 A=u \in Z_{n}^{\times}$and $1-2 B=u^{\prime} \in Z_{n}^{\times}$, then $1-2(A+B-2 A B)=u u^{\prime}$. Thus $A, B \in Z_{n}^{\dot{+}}$ implies $A \dot{+} B \in Z_{n}^{\dot{+}}$. In view of the following equalities,

$$
1-2\left(-A(1-2 A)^{-1}\right)=(1-2 A)(1-2 A)^{-1}+2 A(1-2 A)^{-1}=(1-2 A)^{-1},
$$

we infer that if $A \in Z_{n}^{\dot{+}}$, then $-A \in Z_{n}^{\dot{+}}$, and consequently $\mathcal{Z}_{n}$ is a group. 
Observe that if $n=2 k$, then $1-2 k \in Z_{2 k}^{\dot{+}}$; consequently, the set $\{0, k\}$ is a subgroup of the group $\mathcal{Z}_{2 k}^{\dot{+}}$. Let us define

$$
\mathcal{T}_{n}= \begin{cases}\mathcal{Z}_{n}^{\dot{+}} & \text { if } n \text { is odd } \\ \mathcal{Z}_{2 k}^{\dot{+}} /\{0, k\} & \text { if } n=2 k .\end{cases}
$$

Let us put $\mathcal{T}_{n}=\left(T_{n} ; \dot{+}, \dot{-}, 0\right)$. Now we are able to prove our main result.

Theorem 5.2. For $n \geq 3$, the weak automorphism group $W A U T\left(\mathcal{D}_{n}\right)$ of the dihedral group $\mathcal{D}_{n}$ is a semidirect product of the automorphism group $\operatorname{AUT}\left(\mathcal{D}_{n}\right)$ of $\mathcal{D}_{n}$ and the group $\mathcal{W}_{n}=\left\{\tau_{A, 1,0}: A \in T_{n}\right\}$. A weak automorphism $\tau_{A, a, b}$ is an automorphism of $\mathcal{D}_{n}$ if and only if $A=0$.

Proof. In Theorem 4.2 we have established that

$$
W A U T\left(\mathcal{D}_{n}\right)=\left\{\tau_{A, a, b}: A \in Z_{n}^{\dot{+}}, a \in Z_{n}^{\times}, b \in Z_{n}\right\} .
$$

The weak automorphism $\tau_{A, a, b}$ is the identity permutation if and only if $a i \equiv 0$ $(\bmod n)$ for all $i \in Z_{n}$ and $b+a(1-2 A) j \equiv j(\bmod n)$ for all $j \in Z_{n}$. This is equivalent to the statement $2 A \equiv a-1 \equiv b \equiv 0(\bmod n)$. Therefore the mapping $\varphi$ defined by $\varphi\left(\tau_{A, a, b}\right)=A$ is a surjection of $W A U T\left(\mathcal{D}_{n}\right)$ onto the group $\mathcal{W}_{n}$. Lemma 4.3 shows that $\varphi$ is a homomorphism. From Theorem 4.1, we infer that $\tau_{A, b, a}$ is an automorphism of $\mathcal{D}_{n}$ if and only if $A=0$. Since $\operatorname{AUT}\left(\mathcal{D}_{n}\right)$ is a normal subgroup of $\operatorname{WAUT}\left(\mathcal{D}_{n}\right)$, the proof is complete.

\section{References}

[1] Goetz, A.: On weak automorphisms and weak homomorphisms of abstract algebras. Colloq. Math. 14, 163-167 (1965)

[2] Głazek, K.: On weak automorphisms of finite fields. Coll. Math. Soc. J. Bolyai. 28, 275-300 (1981)

[3] Głazek, K.: Morphisms of general algebras without fixed fundamental operations. In: General Algebra and Applications pp. 89-112. Heldermann Verlag, Berlin (1993)

[4] Higman, G., Neumann, B.H.: Groups as grupoids with one law. Publ. Math. Debrecen 2, 215-221 (1952)

[5] Hulanicki, A., Świerczkowski, S.: On group operations other than $x y$ or $y x$. Publ. Math. Debrecen 9, 142-148 (1962)

[6] Neumann, H.: On a question of Kertesz. Publ. Math. Debrecen 8, 75-78 (1951)

[7] Płonka, E.: On weak automorphisms of algebras having a basis. Colloq. Math. 24, 7-10 (1971)

[8] Płonka, E.: Structure of weak automorphism groups of mono-unary algebras. Algebra Universalis 42, 1-7 (1999)

[9] Solecki, A.: On group operations in groups of exponent k. Colloq. Math. 30, 99-120 (1974)

[10] Streat, A. P.: Subgroups - determining functions on groups. Illinois J. Math. 12, 99-120 (1968)

[11] Szabó, L.: Algebras that are simple with weak automorphisms. Algebra Universalis 42, 205-233 (1999)

[12] Szabó, L.: Infinite algebras with 3-transitive groups of weak automorphisms. Arch. Math. (Brno) 37, 245-256 (2001) 
[13] Traczyk, T.: Weak automorphisms of Boolean and Post algebras. Coll. Math. 13, 159-164 (1965)

[14] Żabka, M.: Weak automorphisms of the permutation group $S_{n}$. Publ. Math. Debrecen 43, 1-8 (1993)

\section{ERNEST PŁONKA}

Institute of Mathematics, The Silesian University of Technology, ul. Akademicka 2a, 44-100 Gliwice, Poland

e-mail: ernest.plonka@polsl.pl

Open Access This article is distributed under the terms of the Creative Commons Attribution Noncommercial License which permits any noncommercial use, distribution, and reproduction in any medium, provided the original author(s) and source are credited. 